\title{
Time-Resolved High-Resolution Angiography Combining Arterial Spin Labeling and Time-of-Flight Imaging
}

\author{
Thomas Lindner $^{1,2}$ (D) Olav Jansen ${ }^{2} \cdot$ Michael Helle $^{3}$
}

Received: 6 May 2020 / Revised: 20 July 2020 / Accepted: 18 September 2020 /

Published online: 30 September 2020

(c) The Author(s) 2020

\begin{abstract}
A strategy to combine two non-contrast-enhanced magnetic resonance angiography techniques is presented. It is based on arterial spin-labeled magnetic resonance imaging to visualize the arterial system at different time points to obtain information about hemodynamic properties in conjunction with a high-resolution time-offlight angiography acquisition. The temporal information obtained by arterial spin labeling (ASL) is combined with the highly spatial resolved time-of-flight image to obtain information about blood flow. Extracting the information of ASL and timeof-flight-imaging leads to images with high spatial resolution which also give information about the temporal course of blood through the intracerebral vasculature. Furthermore, owing to the properties of ASL, visible venous flow in the time-offlight images can be suppressed. The behavior of vascular filling (i.e. signal changes in the ASL) is investigated and used for further interpretation of the data. Furthermore, the ASL data were down-sampled to find a minimally needed spatial resolution to combine both image types. Up to $1.6 \mathrm{~mm}$ isotropic resolution still showed satisfying results rated by two independent readers. In conclusion, a combination of these two different vascular imaging modalities allows to obtain highly spatial and time-resolved images.
\end{abstract}

\section{Introduction}

A detailed visualization of brain feeding arteries and intracranial vessels is important for the diagnosis of many cerebral diseases including stroke, arterio-venous malformations, aneurysms and others [1]. High spatial resolution magnetic resonance

Thomas Lindner

t.lindner@uke.de

1 Department of Diagnostic and Interventional Neuroradiology, University Hospital HamburgEppendorf, Martinistr. 52, 20246 Hamburg, Germany

2 Department of Radiology and Neuroradiology, University Medical Center Schleswig-Holstein, Kiel, Germany

3 Philips GmbH Innovative Technologies, Research Laboratories, Hamburg, Germany 
angiography (MRA) techniques have been developed that allow for the assessment of structural morphology of these vessels. It is, for example, possible to measure the intraluminal diameter in stenotic arteries or to detect small aneurysms [2]. For an advanced diagnosis, however, additional information about the hemodynamics becomes useful [3].

In magnetic resonance imaging (MRI), several acquisition techniques are being used to gather sufficient spatial and temporal information about the cerebral vasculature for a complete diagnosis of the vessel architecture and its hemodynamics [4, 5]. Spatial and temporal information are concluded from different sequences which impedes a correct diagnosis of a variety of diseases, especially when the arteries are altered as in vascular malformations or stroke [6-8]. Furthermore, MRA is an attractive tool for follow-up imaging after interventions to spare patients further exposition of X-rays [9, 10]. The use of dynamic MRA allows the visualization of hemodynamic changes, e.g. in "waitand-see" situations of patients who are not undergoing treatment yet [11]. Thus, combining all information into one image presents relevant information to the radiologist in a concise way for fast and reliable examination of the images.

As MRI, several techniques exist to acquire images of vascular structures and/or hemodynamic properties; the range of eligible sequences is rather high. Still, although each method has its individual benefits, there is not one method that can surpass others and give a comprehensive view of the intracranial vascular situation. Time-of-flight angiography (TOF) is often used in clinical routine measurements as it can generate angiograms with high spatial resolution; however, no hemodynamic information can be gathered [12]. Time-resolved MR methods usually require gadolinium-based contrast agents and only have limited temporal and spatial resolution [13]. Arterial spin labeling (ASL) techniques can create time-resolved angiograms without the usage of contrast agents, but are also limited in spatial resolution to reduce the overall acquisition time which impedes the assessment of small vascular structures [14].

Therefore, a combination of the information of different techniques seems attractive to cancel out individual drawbacks while emphasizing the benefits of each technique and thus simplify an evaluation of the data. In addition, this can also be used to automatically (or semi-automatically) pre-analyze the image information and classify certain properties according to the information of each individual sequence.

The aim of this work is to present a proof-of-concept study that makes it possible to generate angiographic images of the arterial vasculature with high spatial and temporal resolution by combining information of highly spatial resolved TOF acquisitions with the temporal information of ASL images. To reduce the additional time needed as much as possible, it was further checked how low the spatial resolution of ASL can be to still obtain reliable images.

\section{Materials and Methods}

In the present study, 15 healthy volunteers (10 male, 5 female, mean age $27.3 \pm 5.3$ years) underwent MRI scanning under the general protocol for MRI pulse programming approved by the local ethics committee. All volunteers gave written informed consent. 
In the MRI protocol, a TOF angiogram alongside an ASL angiogram was acquired with the same field-of-view (FoV) and geometrical placement.

Image acquisition parameters for ASL were 3D pseudo-continuous Arterial Spin Labeling (pCASL) with a labeling duration of $300 \mathrm{~ms}$ and no post-labeling delay, a temporal resolution of $120 \mathrm{~ms}, 120$ slices with an in-plane voxel size of $1 \times 1 \mathrm{~mm}^{2}$ and $1 \mathrm{~mm}$ slice thickness; T1-Turbo Field Echo (TFE) scan with a TFE factor of 16, SENSE factor: 3, TR/TE: $7.7 / 3.7 \mathrm{~ms}$, flip angle: $10^{\circ}$, half scan factor: 0.7 , resulting in 5 min scan time.

TOF parameters: 171 slices with voxel size $0.41 \times 0.41 \times 0.70 \mathrm{~mm}^{3}$. Fast Field Echo (FFE) Scan with a SENSE factor: 2, TR/TE: 20/3.45 ms, flip angle: $20^{\circ}$, resulting in 6:39 min scan time.

All imaging experiments have been conducted on a Philips Achieva 3T MRI Scanner using a 32-channel receive head coil (Philips Healthcare, Best, The Netherlands).

To match the two images, image reformatting of the ASL images to match the same resolution and slice thickness as the TOF images is required. The workflow is shown in Fig. 1. In this study, this was performed using a tricubic image processing kernel. The images were then registered to counteract subject motion between the individual scans using intensity-based image registration restricted to slice-wise translation and rotation. From the TOF angiogram, a binary mask was created using a Gaussian adaptive thresholding algorithm. This uses the weighted sum of neighborhood values in which the weights are a Gaussian window.

After image pre-processing, the two image types were combined (Fig. 2). On a voxel-by-voxel basis, each time frame of the ASL was combined with the previously created binary mask of the TOF angiogram using a Boolean AND operator. The resulting images then have valid signal only when the masked TOF image has a

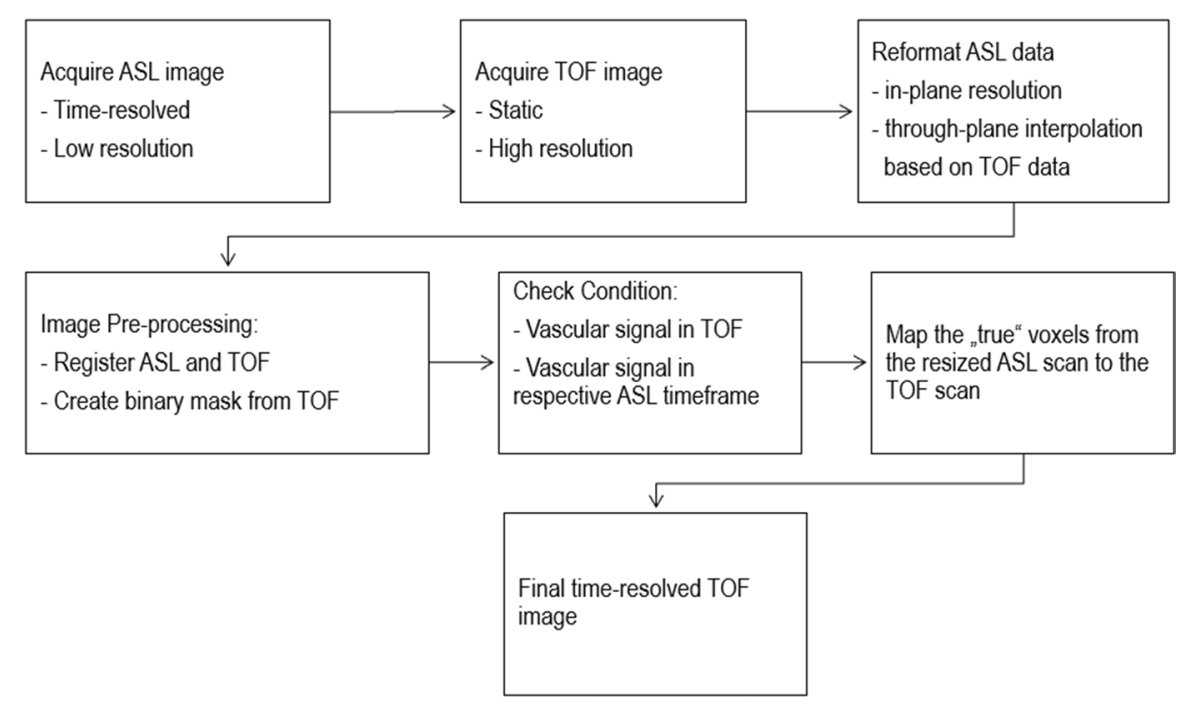

Fig. 1 Flowchart presenting the processing steps to create a time-resolved time-of-flight angiogram 
value of 1 and the ASL image any value larger than 0. Examples of the signal curves are shown in Fig. 3.

The spatial resolution of the source ASL images was additionally reduced stepby-step up to $2 \times 2 \times 2 \mathrm{~mm}^{3}$ with $0.2 \mathrm{~mm}$ increments in all axes to find a cut-off value after which the resulting image still shows sufficient quality. This was assessed by two independent readers (radiologists with 4 and 6 years of experience) blinded to the spatial resolution of the ASL images that were used to create the final images. Image quality was assessed as "overall quality" rather than picking individual arteries. Image quality was assessed using a 4-point grading scale in which 0 would mean "failed image presentation", 1 indicates an excellent image, 2 a sufficient and 3 an unusable image to perform reading on.

\section{Results}

The following signal behavior in the images was observed:

Fig. 2 Resulting images of two selected time points (120 and $480 \mathrm{~ms}$ after labeling) in direct comparison of the arterial spin labeling angiograms (left) with the combined images (right). The first row shows the unaltered time-of-flight angiogram

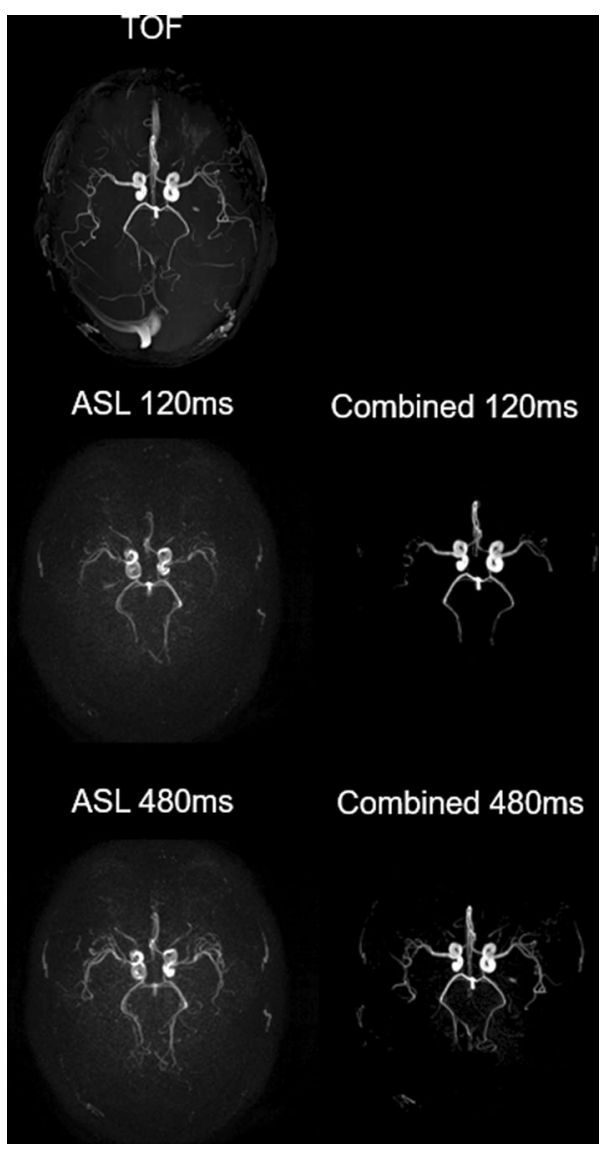




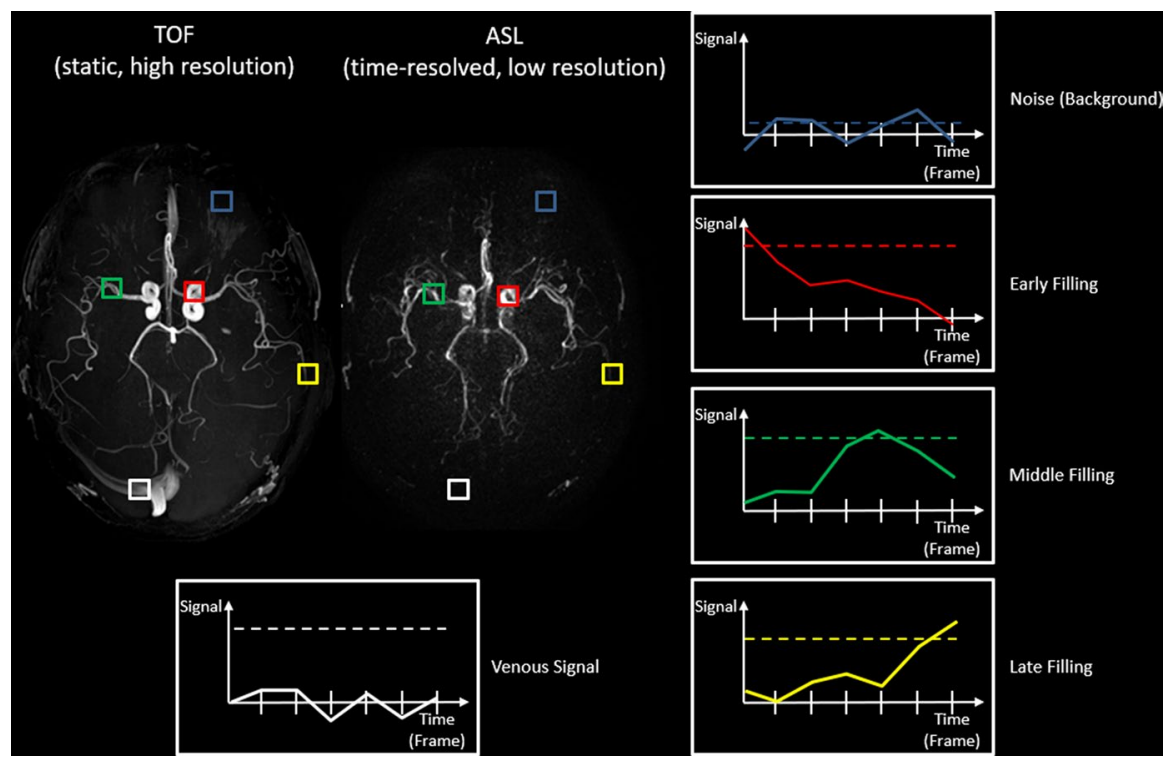

Fig. 3 Different cases of vascular signal shown on the TOF (left) and ASL (right, one time point) scan. Signal is in arbitrary units (a.u.). The blue box shows noise (background) only, while the red box shows an early filled artery, the green box a "middle" filling and the yellow box a late filling peripheral artery. The white box shows venous signal. ASL signal is shown as solid line, TOF signal as dashed

Background signal: in both images, no steady signal can be identified and the signal level remains around zero (positive and negative values). These voxels are, therefore, considered as background signal (Fig. 3, blue box).

Early filling: a vessel is visible in the TOF image and has a certain signal level above the noise level. The ASL signal on the same location has high (maximum) signal on the first temporal phase and then a continuous and steady decrease in signal across the temporal phases. This indicates an early filled artery. As the signal is above the noise level in both acquisitions, the artery can be considered as "true" signal (Fig. 3, red box).

Middle filling: a vessel is visible in the TOF image and the signal level is above the noise level determined by the adaptive thresholding. The ASL signal on the same location has low to zero signal on the first temporal phase and then an increase during the middle temporal time points followed by a decrease in signal towards the last temporal phases. This indicates an early filled artery. As the signal is above the noise level in both acquisitions, the artery can be considered as "true" signal (Fig. 3, red box).

Late filling: a vessel is visible in the TOF image and has a certain signal level above the noise level. The ASL signal on the same location shows a steady increase in signal across the temporal phases. This indicates a late filling artery. As the signal is above the noise level in both acquisitions, the artery can be considered as "true" signal (Fig. 3, red and yellow boxes). 
Venous signal: a vessel is visible in the TOF image and has a certain signal level above noise level. The signal level of the ASL scan fluctuates around zero with irregular shape and no steady change of signal levels. This indicates a venous structure (sinus) and is, therefore, discarded (Fig. 3, blue and green boxes).

The retrospective manipulation of the ASL images' resolution showed that up to $1.6 \times 1.6 \times 1.6 \mathrm{~mm}^{3}$ satisfying results could be obtained (Table 1). With lower spatial resolution, the images were unusable for further diagnosis (Fig. 4). The detailed results of the readers are shown in Table 1, the intraclass correlation coefficient (ICC) between the two readers is 0.405 and the Pearsons' correlation coefficient 0.25 .

\section{Discussion}

This work presents an approach to overcome some problems of angiographic imaging in MRI. First of all, non-contrast-enhanced time-resolved ASL angiographic images generally present a low spatial resolution compared to clinically used static MR angiographic sequences to acquire the images in feasible scan times [14]. The static TOF images on the other hand present a very high spatial resolution, but are not time-resolved, thus, no or only little conclusions about blood flow behavior can be drawn. Image registration is often impeded due to the different spatial resolution of the images and also the low SNR of time-resolved ASL acquisitions.

This study thus was aimed to provide a method to combine the advantages of both techniques to obtain both temporal resolved and highly spatial resolved images. Using images with $1 \mathrm{~mm}^{3}$ isotropic resolution for the ASL, very good results could be obtained when combining ASL and TOF.

The final images (time-resolved TOF) can then be visualized as either dynamic sequence or as time-of-arrival map, meaning that each temporal phase is assigned a different color to visualize inflow properties on a static image [15]. For an automated evaluation of the vascular integrity, the output can be a probability map, meaning that areas of abnormal flow behavior are highlighted on the final images [16]. In the pathological (abnormal) case, the abovementioned conditions might not apply and therefore - on the location of the abnormality - unexpected signal behavior can occur, which would need intervention by a radiologist.

However, acquiring both datasets would increase the scan time to more than 10 min, effectively doubling the scan time of the angiographic acquisition within the scan protocol. Thereby, future improvements should aim for accelerating the ASL scan, e.g. by further reducing the spatial resolution or via more advanced techniques like view sharing or compressed sensing [17].

As selective angiographic imaging (i.e. visualization of a single artery) is possible using ASL, the information from a single artery can be combined with TOF images similarly [18]. As the information of a single artery from ASL is combined with the TOF, the remaining arteries are simply not visible, as no signal is obtained from the selective ASL data on these positions (i.e. signal in TOF "yes", signal in ASL "no", leads to discarding of the voxels). This has the advantage 


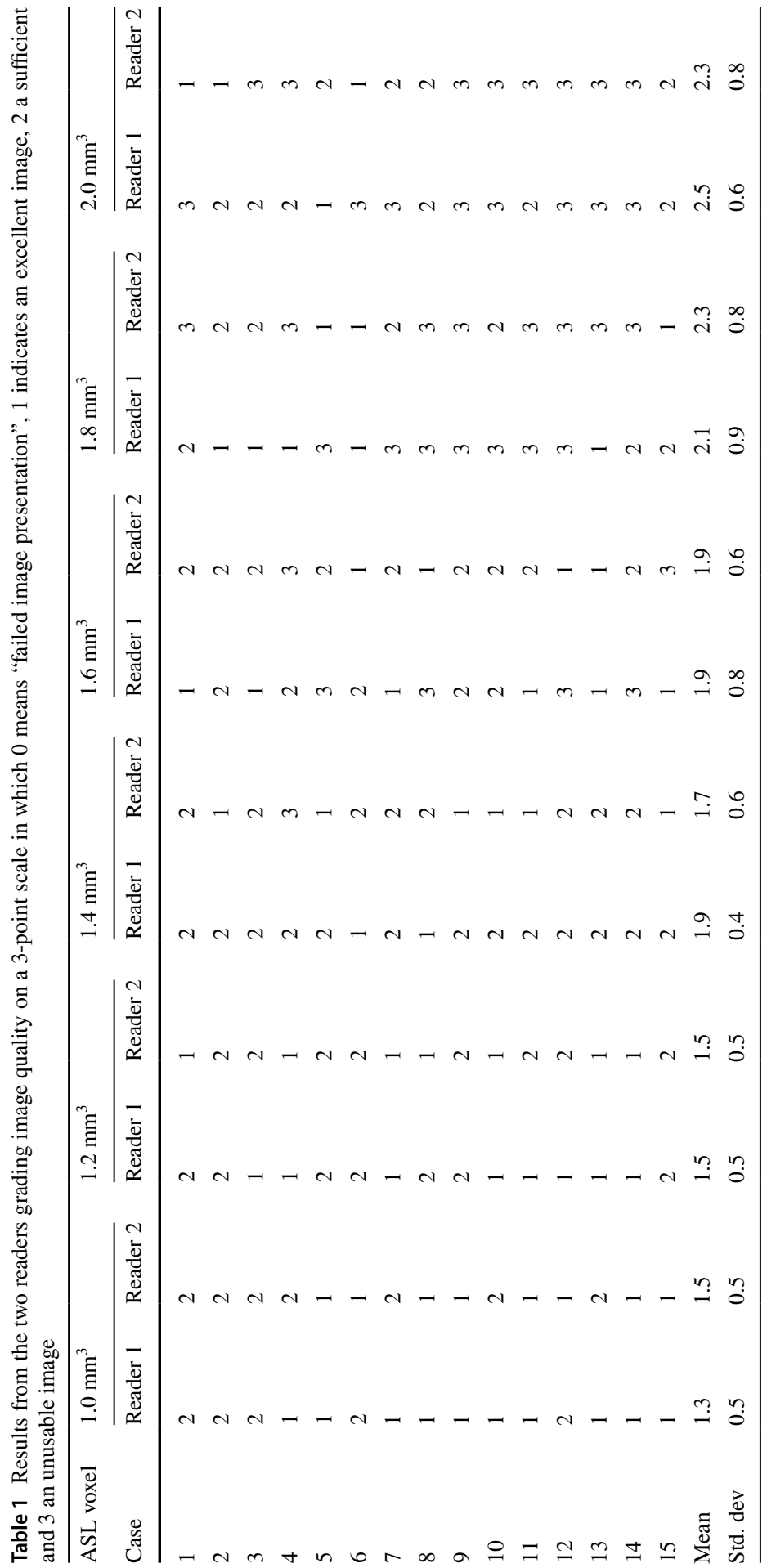




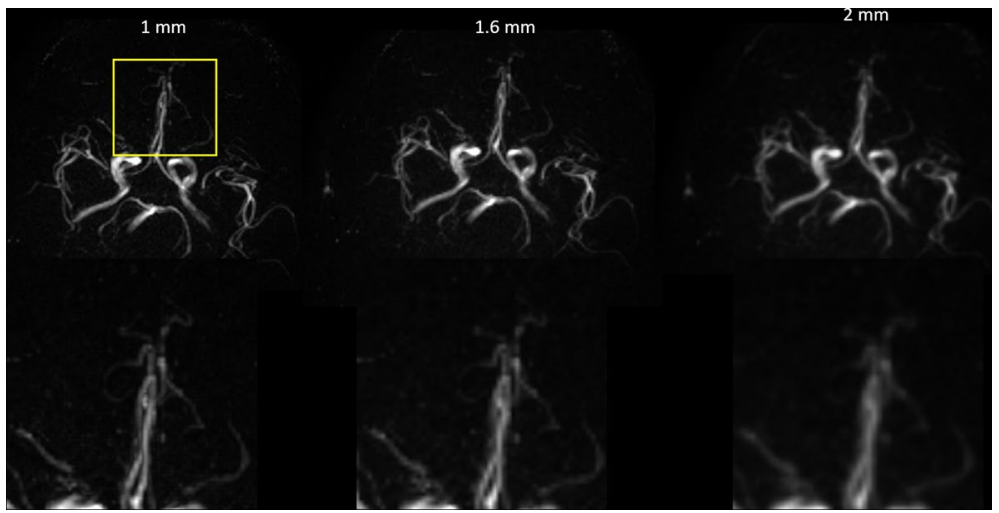

Fig. 4 Comparison between the used 1, 1.6 and $2 \mathrm{~mm}$ resolution ASL images after being combined with the TOF angiogram (i.e. the finally combined images) at one time point after labeling. The upper row shows the whole vasculature, the lower a zoomed-in area of the anterior arteries (indicated in the yellow box). In the case of using the $1 \mathrm{~mm}$ ASL input, the arteries can be easily separated which is still possible with less accuracy at $1.6 \mathrm{~mm}$. In the case of a $2 \mathrm{~mm}$ ASL input image, no distinction can be made anymore. Note that the images in the lower row have been zoomed using a bicubic interpolation for the purpose of preparing the images for display

of drawing conclusions about an individual artery, while the information of the remaining arteries from the TOF data is not lost.

The presented method clearly benefits from applications of machine learning algorithms to predict the possibility of abnormal flow behavior and/or an abnormal vascular situation [19].

Examples include prior segmentation of the TOF and/or ASL dataset to enhance the vessel-to-background contrast, centerline and bifurcation prediction of the vessels, automated detection of impaired flow behavior (e.g. in stenosis) and risk of rupture in aneurysms [20-24]. Additionally, databases of normal vascular images as well as normal flow behavior can be used to early detect pathological changes.

Information about flow behavior (e.g. after stroke treatment) can also be used as a marker for predicting short- or long-term clinical outcome, which has already been proven in a previous study comparing dynamic angiography with static TOF imaging [25].

Acquiring more time points of the ASL data would lead to images of finer temporal resolution. The presented method is not limited to ASL as dynamic scan method, but can also be used to combine, e.g. phase-contrast angiography to TOF or phase-contrast angiography with ASL, leading to more advanced possibilities of interpretation.

Clinical applications include cerebrovascular diseases with complex and diffuse flow patterns, for which not only high-resolution information about the arteries is important, but also underlying hemodynamic properties. These are especially arterio-venous malformations (AVM), but also fistulas, shunting arteries and tumor feeding arteries. Other applications include stenotic arteries, potentially leading to stroke. 
The presented method is, furthermore, not necessarily limited to the cerebral vasculature, but might also be used to visualize other arteries. These include visualization of the renal arteries, the coronary arteries, as well as the peripheral lower leg arteries.

Author Contributions TL: MRI experiments, image post-processing, and ethics considerations. OJ: manuscript preparation and study design. MH: manuscript proofing and study design.

Funding Open Access funding enabled and organized by Projekt DEAL. This study was funded by the German Research Foundation (DFG) under the Grant number JA 875/4-1.

Availability of Data and Material The data are available in anonymized form upon reasonable request.

Code Availability The used code for image processing is available upon reasonable request.

\section{Compliance with Ethical Standards}

Conflict of interest Michael Helle was employed at Philips Research Department, Hamburg, Germany, during the conduction of this study.

Ethics approval The study was approved by the local ethics committee of the medical faculty of the Christian-Albrechts-University Kiel.

Consent to participate All participants gave written informed consent.

Consent for publication All authors give consent to publish this manuscript.

Open Access This article is licensed under a Creative Commons Attribution 4.0 International License, which permits use, sharing, adaptation, distribution and reproduction in any medium or format, as long as you give appropriate credit to the original author(s) and the source, provide a link to the Creative Commons licence, and indicate if changes were made. The images or other third party material in this article are included in the article's Creative Commons licence, unless indicated otherwise in a credit line to the material. If material is not included in the article's Creative Commons licence and your intended use is not permitted by statutory regulation or exceeds the permitted use, you will need to obtain permission directly from the copyright holder. To view a copy of this licence, visit http://creativecommons.org/licen ses/by/4.0\%.

\section{References}

1. M.L. Portegies, P.J. Koudstaal, M.A. Ikram, Handb. Clin. Neurol. 138, 239-261 (2016)

2. A.J.M. Kiruluta, R.G. González, Handb. Clin. Neurol. 135, 137-149 (2016)

3. D.S. Liebeskind, N. Sanossian, Neurology 79(13 Suppl 1), S105-S109 (2012)

4. J. Wikström, E. Ronne-Engström, G. Gal, P. Enblad, M. Tovi, Acta Radiol. 49(2), 190-196 (2008)

5. E. Maj, A. Cieszanowski, O. Rowiński, M. Wojtaszek, M. Szostek, R. Tworus, Pol. J. Radiol. 75(1), 52-60 (2010)

6. C.S. van Rijswijk, E. van der Linden, H.J. van der Woude, J.M. van Baalen, J.L. Bloem, AJR Am. J. Roentgenol. 178(5), 1181-1187 (2002)

7. A. Le Bras, H. Raoult, J.C. Ferré, T. Ronzière, J.Y. Gauvrit, AJNR Am. J. Neuroradiol. 36(6), 1081$1088(2015)$

8. T. Boujan, U. Neuberger, J. Pfaff et al., AJNR Am. J. Neuroradiol. 39(9), 1710-1716 (2018) 
9. C. Timsit, S. Soize, A. Benaissa, C. Portefaix, J.Y. Gauvrit, L. Pierot, AJNR Am. J. Neuroradiol. 37(9), 1684-1689 (2016)

10. M.J. van der Laan, C.J. Bakker, J.D. Blankensteijn, L.W. Bartels, Eur. J. Vasc. Endovasc. Surg. 31(2), 130-135 (2006)

11. M. Trojan, F. Rengier, D. Kotelis, M. Müller-Eschner, S. Partovi, C. Fink, C. Karmonik, D. Böckler, H.U. Kauczor, H. von Tengg-Kobligk, Contrast Media Mol. Imaging. 2017, 5428914 (2017)

12. M. Cirillo, F. Scomazzoni, L. Cirillo, M. Cadioli, F. Simionato, A. Iadanza, M. Kirchin, C. Righi, N. Anzalone, Eur. J. Radiol. 82(12), e853-e859 (2013)

13. D.R. Hadizadeh, C. Marx, J. Gieseke, H.H. Schild, W.A. Willinek, Rofo. 186(9), 847-859 (2014)

14. T. Lindner, U. Jensen-Kondering, M.J. van Osch, O. Jansen, M. Helle, Magn. Reson. Imaging 33(6), 840-846 (2015)

15. S.J. Riederer, C.R. Haider, E.A. Borisch, Radiology 253(2), 532-542 (2009)

16. F. Hassainia, D. Petit, J. Montplaisir, Brain Topogr. 7(1), 3-8 (1994)

17. E. Levine, B. Daniel, S. Vasanawala, B. Hargreaves, M. Saranathan, Magn. Reson. Med. 77(5), 1774-1785 (2017)

18. U. Jensen-Kondering, T. Lindner, M.J. van Osch, A. Rohr, O. Jansen, M. Helle, Eur. J. Radiol. 84(9), 1758-1767 (2015)

19. R.C. Deo, Circulation 132(20), 1920-1930 (2015)

20. R. Phellan, N.D. Forkert, Med. Phys. 44(11), 5901-5915 (2017)

21. G. Tetteh, V. Efremov, N.D. Forkert, M. Schneider, J. Kirschke, B. Weber, C. Zimmer, M. Piraud, B.H. Menze, DeepVesselNet: vessel segmentation, centerline prediction, and bifurcation detection in 3-D angiographic volumes. https://arxiv.org/ftp/arxiv/papers/1803/1803.09340.pdf

22. M. Livne, J. Rieger, O.U. Aydin, A.A. Taha, E.M. Akay, T. Kossen, J. Sobesky, J.D. Kelleher, K. Hildebrand, D. Frey, V.I. Madai, Front. Neurosci. 13, 97 (2019)

23. T. Canchi, S.D. Kumar, E.Y. Ng, S. Narayanan, Biomed. Res. Int. 2015, 861627 (2015)

24. S.L. Waddle, M.R. Juttukonda, S.K. Lants, L.T. Davis, R. Chitale, M.R. Fusco, L.C. Jordan, M.J. Donahue, J. Cereb. Blood Flow Metab. 40(4), 705-719 (2020)

25. T. Boujan, U. Neuberger, U. Pfaff, S. Nagel, C. Herweh, M. Bendszus, M.A. Möhlenbruch, AJNR 39(9), 1710-1716 (2018)

Publisher's Note Springer Nature remains neutral with regard to jurisdictional claims in published maps and institutional affiliations. 